\title{
Aristofane nel Cornu Copiae di Niccolò Perotti
}

\author{
Ludovica Radif
}

(Palacký University in Olomouc)

\section{Aristophanes in Niccolò Perotti's Cornu Copiae}

\begin{abstract}
The aims of this paper are to analyse the quotations and references in Perotti's Cornu Copiae to the ancient comedy of Aristophanes and to understand the context in which ancient words are considered by the humanist. Their role seems (as usually the whole aim of that work) simply auxiliary in order to give some semantic information about Latin expressions. But, as with the references to the Plutus, we can hypothesize direct knowledge of the text.
\end{abstract}

\section{Keywords}

Aristophanes in the 15th century; Plutus in the Renaissance; Perotti's Cornu Copiae 
Nell'ambito della ripresa umanistica di Aristofane ritroviamo il nome dello studioso inglese William Gray, in quanto, divenuto vescovo di Ely, lascia poi in eredità al Balliol College, luogo della sua formazione, molti manoscritti, compreso l'unico testimone della fabula Penia di Rinuccio Aretino, prima traduzione parziale del Pluto aristofaneo. ${ }^{1}$ Colui che più tardi ha l'onore di ricevere appunto il testo da Rinuccio, durante i suoi viaggi di studio vive in Italia, e a Ferrara incontra e si trova ad accogliere in casa propria Niccolò Perotti (intorno al 1446): questi, giovanissimo, lo aiuta nell'apprendimento della lingua greca, appresa a Mantova con Vittorino da Feltre e poi a Ferrara probabilmente con Guarino da Verona. ${ }^{2}$

Anche il Perotti gioca una piccola parte nella fortuna di Aristofane nel Quattrocento, come è possibile vedere analizzando in particolare il suo Cornu Copiae (CC), il commento a Marziale, opera monumentale corrispondente a un'enciclopedia, dove è possibile rintracciare l'intera cultura della sua lunga vita di studioso. ${ }^{3}$ In un recente articolo espressamente dedicato alla cultura greca di Niccolò Perotti, Jean-Louis Charlet ha posto in evidenza tutti i riferimenti presenti nel Cornu Copiae ad autori greci. ${ }^{4}$ Il loro numero rilevante (circa 800, di cui 107 in lingua greca), considerato insieme alla varietà delle fonti, testimonia la complessa rete di testi di cui l'umanista si avvaleva nel dichiarato tentativo di restituire al latino la sua grandezza. Il paragrafo che riguarda le riprese da Aristofane (270-271) mi ha suggerito un percorso specifico volto a esplorare se si tratti di rinvii solo occasionali o se possano essere considerati segno di un qualche suo interesse per il comico antico. Si tratta quindi anzitutto di esaminare i dodici rinvii presenti nel Cornu Copiae, ricercando le possibili implicazioni della ripresa di determinati termini o passaggi delle commedie, della loro traduzione o contestualizzazione. Fin da subito possiamo ritenere in ogni caso preziosa l'analisi dei passi perottini in quanto sono pur sempre un documento relativo ad Aristofane nel Quattrocento, epoca in cui, non essendo ancora stato edito a stampa il patrimonio comico arcaico risulta ancora poco conosciuto e scarsamente accessibile anche ai dotti. ${ }^{5}$ Riporterò dunque i passaggi del Cornu Copiae contenenti i rinvii ad Ari-

1 Oxford, Balliol College, ms. Lat. 131, ff. 31r-37r. Ludwig (1975); Radif (2011). Composta già negli anni attorno al 1415, fu probabilmente rimaneggiata e rivista nella piena maturità: Pillolla (1993: pp. 42-43).

2 Guarino aveva avuto come maestro a Costantinopoli Emanuele Crisolora, cui era succeduto nello Studio Fiorentino; come, nel dibattito al riguardo, ha avuto occasione di sottolineare piú di una volta J.-L. Charlet introducendo la figura del Perotti, il suo soggiorno, anche dopo la dipartita di Vittorino, nella città di Ferrara, dove Guarino teneva scuola dal 1429, lascia presumere altamente probabile la sua frequentazione di Guarino, che troverebbe una conferma nel suo rimanere in contatto con altri allievi di Guarino, quali, per esempio, Giano Pannonio.

3 La più seria attenzione filologica al testo, relativamente recente, a partire dagli anni '80 del Novecento, prima dei quali possiamo ricordare: Sabbadini (1922), Mercati (1946), Weiss (1964; 1969), ha favorito molti contributi sull'attività e sullo stile dell'Umanista e portato all'attuale edizione collettiva del Cornu Copiae, diretta da J.-L. Charlet: Charlet (1989-2001). Per lo sviluppo della letteratura sull'argomento si vedano gli articoli: Prete $(1980 ; 1986 ; 1990)$ e l'assai ricca produzione di Jean-Louis Charlet, tra cui: Charlet (1990; 1994; 2009; 2011a; 2011b).

4 Charlet (2013).

5 Ricordiamo infatti che l'editio princeps (contenente nove commedie) fu realizzata da Aldo Manuzio a Venezia nel 1498, mentre le due rimanenti commedie, Tesmoforiazuse e Lisistrata, vennero stampate solo nel 1515 a Firenze per i tipi dei Giunti. 
stofane e di seguito le fonti e i luoghi eventuali delle commedie cui si accenna. Il numero complessivo è di dodici, se eliminiamo il rinvio erroneo ad Aristofane a proposito di una citazione in greco riguardo agli enigmi (CC 12, 35, 3-4), che appartiene in realtà a Sofocle (Oed. T. 439; preso dalla Suda, seguito da traduzione latina).

Questo in sintesi lo schema delle riprese, che, nell'ordine di esposizione dei passi del Cornu Copiae, tiene conto della gradualità decrescente di approccio al testo aristofaneo. Il primo gruppo riunisce rinvii precisi ad Aristofane, a volte scritti in greco, per i quali è possibile operare un confronto diretto con alcuni passi e, in alcuni casi, indicare probabili fonti corrispondenti, che generalmente sono state già individuate e indicate nell'apparato relativo alle fonti dell'edizione critica di riferimento. Il secondo gruppo accoglie i termini ricondotti ad Aristofane, per i quali abbiamo notizie frammentarie. Il terzo gruppo riunisce le espressioni proverbiali, non direttamente attribuite ad Aristofane, ma che effettivamente sono presenti, in qualche modo, anche nell'opera aristofanea. I GRUPPO:

1- Uccelli testo greco [Suda] aggiunta traduzione CC 37, 5, 9-10

2- Pluto testo greco [Suda] aggiunta traduzione CG 28, 37, 8

3- Pluto testo greco [?] CC 28, 37, 10

4- Pluto testo greco [Suda] CC 2, 526, 13-14

5- Rane testo greco [Gellio] aggiunta traduzione CC 1, 390, 7-12

6- Acarnesi passo in traduzione [Plinio] CC 1, 6, 11-13

7- Acarnesi passo in traduzione [Plinio] CC 2, 696, 7-8

II GRUPPO:

8- Ar. Fr. Vocabolo traslitterato "Afrodite" [Macrobio] CC 3, 124, 12

9- Ar. Fr. Vocabolo traslitterato "Baccar" [Plinio] CC 12, 93, 16-17

III GRUPPO:

10- Ar. Fr. Proverbio generico "Corinto" [Gellio] CC 6, 402, 4-6

11- Proverbio generico "Eracle" (Lisistrata) CC 6, 339, 3

12- Riferimento generico ai misteri (Tesmoforiazuse) CC 6, 262, 2-4

\section{1- CC. 37, 5, 9-10}

...Idem parochos, inquit, hoc est idem auspex sive paranymphus. Hunc enim mos erat preire vehiculum, in quo sponsus ac sponsa ferebantur, quo die nuptiae celebrabantur. Aristophanes:

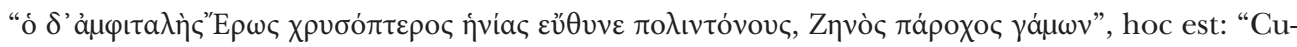
pido floridus aureis praeditus alis habenas dirigebat aversas parochus nuptiarum Iovis".

E questi dice parochos, cioè auspice o paraninfo. La tradizione voleva che costui aprisse la strada al carro, in cui venivano portati lo sposo e la sposa, nel giorno in cui si celebravano le nozze. Aristofane... ${ }^{6}$

Suid. s.v. Пápoхоc: П 710

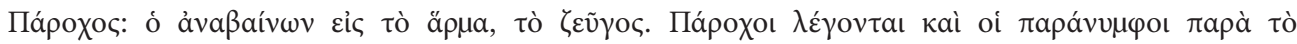

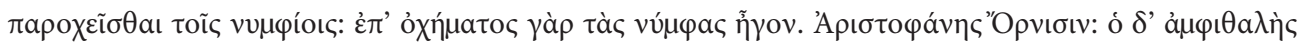

6 Traduzioni mie. 


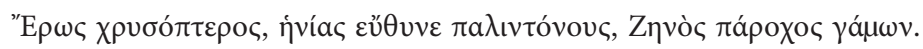

Parochos: colui che sale sul carro, trainato da cavalli. Sono detti parochoi anche i paraninfi, a motivo della loro corsa (parocheisthai) al fianco degli sposi: erano soliti condurre le spose sul carro. Così Aristofane negli Uccelli...

Il riferimento è a Uccelli 1737-1740, quando, durante il festeggiamento di quelle nozze di respiro intermondiale che abbracciano le stirpi celesti degli dèi, degli uomini e degli uccelli, tra Regina e Pistetero, il coro così prorompe:

Ar. Av. $1737-1740$

Xopóc

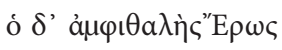

Хрvбó $\tau \varepsilon \rho \rho \varsigma$ ìvía

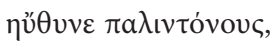

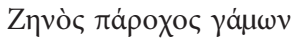

Eros fiorente dall'ali dorate

teneva le redini tese,

pronubo custode

delle nozze di Zeus

Perotti si richiama alla voce della Suda a proposito del termine parochos, dalla quale infatti, viene ripreso esattamente lo stesso numero di parole, eccetto l'indicazione del titolo della commedia Uccelli, che egli omette, operando come di consueto nel caso di citazione da altri autori.

\section{2/3- CC 28, 37, 8-10}

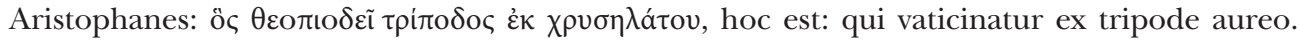

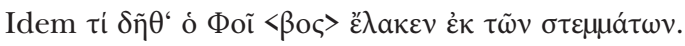

Aristofane: il quale sforna oracoli dal tripode dorato: cioè che vaticina dal tripode aureo.

E lo stesso: che dunque ha vaticinato Febo dalle sue bende?

Suid. s.v. $\Theta \varepsilon \sigma \pi \iota \omega \delta \varepsilon \tilde{\varepsilon}: \Theta 281$

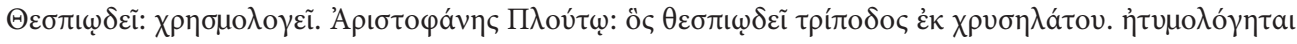

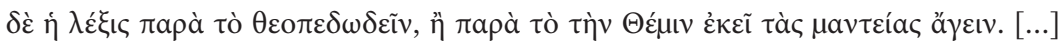

Thespiodei: dà oracoli. Aristofane nel Pluto... Il termine trova origine nel verbo "frequentare un dio" (theopedodein) o dal fatto che Temi porta lì le sue profezie.

\section{Ar. $P l .8-9$}

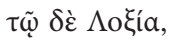

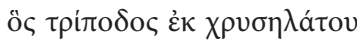

ma mi irrita il Lossia (Apollo),

che dal suo tripode dorato... 
Il servo Carione, dopo essersi lamentato della situazione assurda in cui si trova, scherza riguardo al potere dell'oracolo di Apollo; poco più avanti chiede dunque al padrone che cosa abbia vaticinato il grande Febo.

Ar. $P l .39$

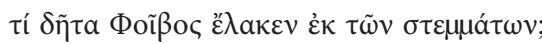

che dunque ha vaticinato Febo dalle sue bende?

Parlando di Delfi, collocata sulle pendici del monte Parnaso, e del tempio di Apollo, nota come non fossero muri a difenderla, ma i precipizi, che ne fanno un luogo quasi altrettanto miracoloso quanto la potenza della divinità ivi espressa, con uno straordinario effetto di moltiplicazione delle voci a eco. Riferisce di come nella pianura si aprisse un foro, dove si trovava il tripode, da cui uno spiritus, levandosi, porgeva alle donne, che da Febo prendevano il nome di Phoebadas, gli oracoli, che quelle immediatamente cantavano in versi. Spiega poi la voce stemmata.

\section{4- CC 2, 526, 13-14}

Quidam tamen existimaverunt delirare a graeco deductum, quasi apud quos $\lambda \eta \rho \varepsilon \tilde{v}$ ineptire significat et $\lambda \tilde{\eta} \rho \circ v$ deliramentum. Aristophanes: $\lambda \tilde{\eta} \rho o v ~ \lambda \eta \rho \varepsilon \tilde{\varsigma}$.

Alcuni tuttavia ritennero che "delirare" derivasse dal greco, per cui lerein significa "impazzire" e leron "delirio".

Suid. s.v. $\Lambda \eta \rho \varepsilon \tilde{\varsigma}: \Lambda 467$

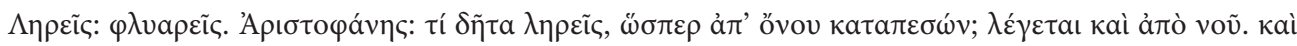

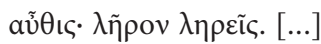

Lereis: "dici sciocchezze". Aristofane: "stai delirando", come uno andato giù dall'asino ( $a p$ onu) fuori strada, intendendo anche "andato fuori di testa" (apo nu). E ancora "stai delirando".

\section{Ar. $P l .517$}

\section{$\mathrm{X} \rho \varepsilon \mu u ́ \lambda o \varsigma$}

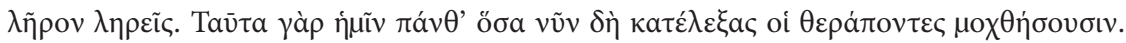

Stai delirando: a tutte queste incombenze che hai elencato adesso

provvederanno i servitori.

In questo passo l'umanista, mentre considera necessario apporre la traduzione della forma verbale indicante l'agire in modo insensato (ineptire) e del corrispondente nome relativo alla sciocchezza (deliramentum), reputa evidentemente superfluo trasportare in latino la breve battuta con cui Cremilo cerca di controbattere le affermazioni di Penia ( $\lambda \tilde{\eta} \rho$ ov $\lambda \eta \rho \varepsilon \tilde{\varsigma})$; probabilmente pensa che il suo lettore sarà in grado di seguirlo nella coniugazione del verbo alla seconda persona singolare.

7 In questo caso il riferimento è alle Nuvole, v. 500. 
Qui l'allusione è precisa: la battuta gioca con la costruzione dell'accusativo dell'oggetto interno, difficile da rendere in italiano, ma indicante il delirare di delirio, ossia il parlare senza senso. L'espressione comica è gestita sulla linea del paradossale: infatti, il personaggio va immaginando soluzioni alle prospettive catastrofiche che gli sta preannunciando la personificazione della povertà e che gli sembrano del tutto assurde.

\section{5- CC 1, 390, 7-12}

Aristophanes poeta facetissimus vastam illam atque inanem hominum insipientium loquacita-

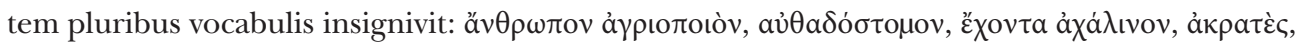

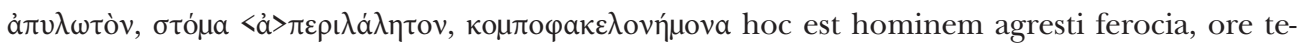
merario, infreni, prodigo, petulante, loquacem, futilem et quadam verborum congerie ac fastu profusum.

Aristofane, poeta assai divertente, ricoprì di definizioni quella sconfinata e vuota loquacità degli uomini stupidi: "bocca sfrenata, plasmatrice di mostri lingua sbrigliata che non conosce barriere, chiacchierone incontinente, millantatore di gonfiaggini”: ossia un uomo che si esprime con villana insolenza, lingua incauta e senza freni, lunga e petulante, frivolo ed esorbitante di un cumulo di espressioni altisonanti.

Gell. 15, 19-20

Huiuscemodi autem loquacitatem verborumque turbam magnitudine inani vastam facetissimus poeta Aristophanes insignibus vocabulis denotavit in his versibus:

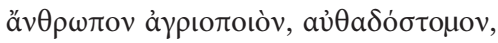

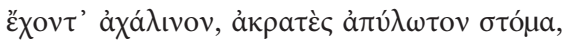

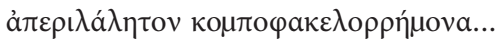

Il poeta Aristofane descrisse questo tipo di loquacità e ampia turba di parole di vuota grandezza nei seguenti versi.

Ar. $R a .836-839$

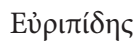

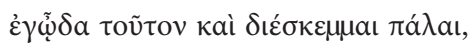

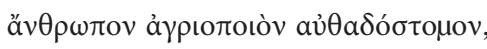

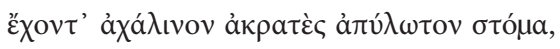

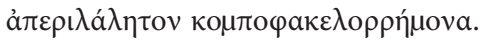

Lo conosco, lo sto scrutando da tempo, questa bocca sfrenata, plasmatrice di mostri, lingua sbrigliata che non conosce barriere, chiacchierone incontinente, millantatore di gonfiaggini.

A proposito del vaniloquio, sostiene che Omero nel riferirsi a Ulisse, fornito di saggia facondia, dicesse che l'eroe emetteva la voce non semplicemente dalla bocca, ma dal petto, indicando con questa immagine una maggiore profondità di concetto al di là del mero atto fisiologico. Parallelamente e in opposizione a questa rappresentazione, la temerarietà delle espressioni non ponderate si visualizza con la metafora delle parole che 
oltrepassano la barriera dei denti. Euripide nelle Rane, precisamente in quel passaggio, sta parlando di Eschilo (nella famosa disputa messa in scena da Aristofane surrettiziamente nell'Ade) e lo definisce così, affermando che Eschilo ha creato personaggi rudi e cocciuti e che inoltre la sua bocca è senza freno, non bloccata, insomma senza controllo.

Parlando di problemi letterari e di ripetizioni, a Gellio non sfugge il richiamo a quella famosa situazione comica:

apud Aristophanem, facetissimum comicorum, Euripides Aeschylum insectatus est cum ait:

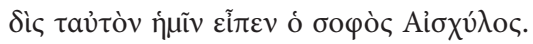

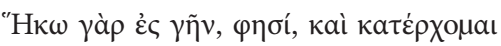

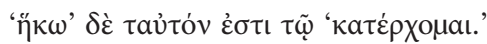

In Aristofane, il più divertente dei comici, Euripide perseguita Eschilo dicendogli:

Il buon Eschilo dice due volte la stessa cosa.

Rientro in questa terra, faccio ritorno.

"Rientro" è la stessa cosa che "faccio ritorno".

Il passo della commedia presenta caratteristiche che ben si adattano a un'indagine lessicografica: quelli che, fatti scendere a cascata contro Eschilo e il suo modo di caratterizzare i personaggi, suonano insulti e per il pubblico risuonano comici, nel contesto si rivelano preziosi sinonimi. Ecco quindi la motivazione della ripresa perottina. In questo caso, egli si limita ad aggiungere la traduzione latina, che in Gellio manca. Va

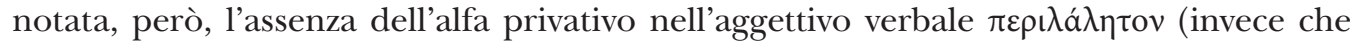
ả $\varepsilon \rho \iota \lambda a ́ \lambda \eta \tau o v)$ per come viene riportato dall'umanista, il quale tuttavia riesce a restituire comunque bene il senso dello smodato impiego di una congerie di paroloni sontuosi.

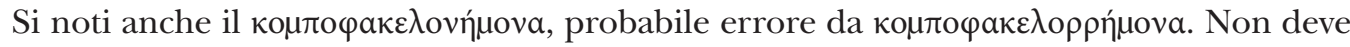
sfuggire tuttavia il dettaglio di quel poeta facetissimus apposizione del nome del Comico, che, pur ripreso pari pari, potrebbe anche tradire un certo compiacimento nei confronti della ricchezza verbale aristofanea.

\section{6- CG 1, 6, 11-13}

...Alii scandicem. ${ }^{8}$ Unde per iocum Aristophanes

Eurypidi poetae obiecit quod mater eius ne olus quidem legitimum venditasset, sed scandicem.

Altri la dicono scandix. Da cui per scherzo Aristofane rinfacciava al poeta

Euripide il fatto che sua madre non vendeva la verdura buona, ma quella selvatica.

\section{Plin. HN. 22, 38}

Haec est quam Aristophanes Euripidi poetae obicit ioculariter, matrem eius ne olus quidem legitimum venditasse, sed scandicem.

Questa è la pianta che Aristofane per scherzo rinfaccia al poeta Euripide quando dice che sua madre non vendeva verdura buona ma quella selvatica.

8 Ricordiamo che l’origine vera del termine è da identificarsi nel gr. $\sigma \chi \alpha \dot{\alpha} \zeta \omega$ "pungo", proprio per la forma allungata dei frutti che sembrano spille o pettini, per cui infatti la pianta è anche chiamata volgarmente "pettine di Venere". 


\title{
Aulus Gellius Noctes Atticae XV, 20, 1-2
}

Euripidi poetae matrem Theopompus agrestia olera vendentem victum quaesisse dicit

Teopompo racconta che la madre di Euripide si guadagnasse da vivere rifilando erbe selvatiche

\author{
Ar. Ach. 478

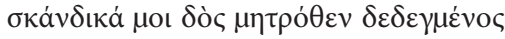 \\ Dammi il cerfoglio ereditato dalla mamma
}

A proposito del termine barba, che non deve ritenersi collegabile a un'usanza dei barbari di portarla particolarmente lunga, il commentatore di Marziale dice che si può riferire sia agli uomini sia ad altre forme animate o inanimate. ${ }^{9}$ Sostiene che i pesci molli presentino due file di barba sul labbro inferiore, che viene detta anche tragopogus, per la somiglianza con quella dell'hircus, il caprone, con rinvio ai due corrispettivi termini greci $\tau \rho \dot{\gamma} \gamma o \varsigma$ e $\pi \dot{\omega} \gamma \omega \nu$. Altri la dicono comen, in quanto analoga alla chioma degli alberi, che sparge le foglie in cerchio; altri ancora scandicem. Proprio su quest'ultimo termine si appunta qui l'attenzione, con un rinvio ad Aristofane, nel suo rinfacciare al poeta Euripide, riprendendolo da Plinio, il fatto che la madre di lui non era solita vendere verdura autentica, di qualità commerciale, ma scandix, cioè cerfoglio, una pianta selvatica, aromatica e medicinale. ${ }^{10}$

Il passo nel contesto è:

Ach. 478-480

$\Delta$ เкаı́то入ıс:

$[\ldots]$

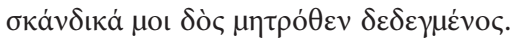

Dammi il cerfoglio ereditato dalla mamma

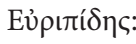

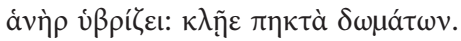

Quest'uomo insolentisce: chiudi le imposte di casa

$\Delta$ เкаı́толфıс:

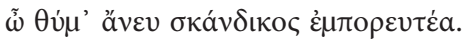

Bisogna, caro mio, andarsene senza il cerfoglio

$[\ldots]$

Quando Diceopoli, il protagonista, chiede scherzosamente a Euripide di dargli un po' del cerfoglio rifilato da sua madre, Euripide reagisce parlando di űßpıৎ "tracotanza", di fatto appunto traducendo in termini di violenza verbale la battuta appena ascoltata. Al

9 Afferma (CC 6, 4 quod scribit Servius barbam hominum, barbas brutorum esse, falsum est). Quello che scrive Servio, che "la barba" è cosa riferita agli uomini, mentre "le barbe" agli animali, è falso. Sull'argomento, in riguardo alla posizione di Lorenzo Valla, cf.: Abbamonte (2011: pp. 471-472).

10 Sulle occorrenze di nomi di piante in Aristofane, si legga: Amigues (1988: pp. 164-165, su scandix). 
di là della credibilità del discusso riferimento alla madre di Euripide come venditrice di verdura, mi pare da sottolineare ciò che forse solitamente nelle moderne traduzioni di Aristofane non sempre viene evidenziato: ossia l'ingiuria legata al tipo di verdura smerciato dalla madre; il punto della satira, che giustifica anche la reazione del poeta, non è tanto il mestiere in sé quanto l'inganno con cui essa lo esercitava ossia rifilando verdura selvatica come la scandice, il cerfoglio, invece della presunta olus verdura in vendita normale. ${ }^{11}$

Ricollegato al medesimo concetto del cerfoglio è anche il passo di CC 2, 696, 7-8, come si vedrà, dove compare il nome scientifico della pianta scandix, della famiglia delle apiacee (per intenderci, la stessa del sedano), detta anche pettine di Venere o spilla di pastore, per la forma appuntita dei suoi frutti, per cui anche si parla di "unghie del diavolo". A. Polito sostiene che tanto il termine scandix in Plinio, quanto il corrispettivo greco, siano impiegati a indicare il cerfoglio; in entrambi i casi, siamo in presenza di un'erba selvatica di uso comune (il cerfoglio, per esempio viene impiegato nella famosa sauce Béarnaise $).{ }^{12}$ La scandice nella tradizione medicinale, come nel trattato di Pietro Mattioli, risulta un'erba selvatica e amara da utilizzarsi nella preparazione di decotti atti a favorire la diuresi. ${ }^{13}$

Anche nelle Tesmoforiazuse abbiamo un rinvio alla questione del mestiere della madre del poeta, quando si afferma che Euripide sia a tal punto "selvatico" nei suoi modi da rendere ben visibile la propria provenienza dalle erbe ... selvatiche:

\section{Ar. Th. 455-456}

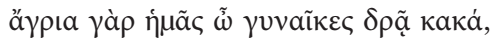

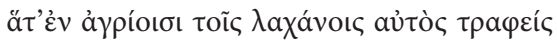
donne, cresciuto tra le erbe selvatiche,
è selvatico e malevolo con noi

Parlando delle erbe che gli Egiziani comunemente consumano, Plinio nomina la scandix, che da altri è chiamata tragopona, caratterizzata da foglie molto simili a quelle del croco, riferendo, come si è visto $(22,38)$ della madre di Euripide, la quale smerciava scandix invece di olus [...] legitimum.

\section{Ar. Th. 387}

Eủ

da Euripide, il figlio dell'erbivendola

L'atteggiamento dell'umanista nei confronti di Plinio è di sostanziale ripresa, che si configura come un elemento di arricchimento a proposito del lemma di volta in volta preso in esame.

11 Parlando delle sottigliezze verbali di Euripide e del loro risvolto nella commedia, fa giustamente riferimento all'interpretazione di Plinio, e quindi all'inganno, Bagordo (2008).

12 Polito (2011).

13 Mattioli (1744: pp. 300-301). Sul termine, si veda anche: André (1985: pp. 229, 301, 326). 
Per quanto riguarda Gellio, attribuisce al retore e storico antico Teopompo alcune notizie sul drammaturgo, tra cui le note umili origini. Questa è la prima frase che lì si sceglie per caratterizzare il poeta, alla quale fa seguito invece un'altra informazione relativa al nobile destino di vincitore nelle gare, a suo tempo comunicata al padre dagli astrologi (2-3).

Proseguendo nella caratterizzazione del tragediografo, Gellio cita una grotta, che egli aveva visto, nella quale pare che Euripide scrivesse tragedie (5); e quanto alla sua scarsa simpatia nei riguardi delle donne, rinvia ai versi delle Tesmoforiazuse che lo dicono cresciuto tra le erbe selvatiche (7).

\section{7- CC 2, 696, 7-8}

... Sunt etiam qui scandicem vocent. Haec est quam Aristophanes Eurypidi poetae obiicit ioculariter, matrem eius ne olus quidem legitimum venditasse, sed scandicem.

C'è chi la chiama scandix. Si tratta della pianta che Aristofane rinfaccia scherzando a Euripide, nel senso che sua madre non vendeva verdura buona, ma della scandice.

A proposito del termine tragus, corrispondente a hircus, tra i derivati ricorda l'uccello tragopa e il tragelaphus, una specie di cervo, ma anche la tragopus, un'erba che da alcuni è denominata barbula hircina, [...] alcuni la chiamano scandix. Quindi il tema è il medesimo e notiamo come lo studioso riprenda nuovamente lo stesso da Plinio, semplicemente adattando la frase da un punto di vista sintattico al suo contesto.

\section{8- CC 3, 124, 12}

Propterea veteres Venerem non deam solum, sed etiam deum nominabant (8-9)

Aristophanes eam non Aphroditen sed Aphroditon nominat

\section{Cfr. Ar. 702 K}

Perciò gli antichi denominavano Venere non solo una dea ma anche un dio.

Parlando di Afrodite e delle tre differenti Veneri, afferma che la propensione ai piaceri di Venere riguarda il genere umano indistintamente, maschi e femmine, per cui gli antichi immaginarono che Venere fosse contemporaneamente uomo e donna e non chiamarono esclusivamente dea Venere, ma anche deus, tanto che a Cipro si dice che la statua avesse il corpo peloso e il vestito muliebre con scettro e statura virile. In realtà, in Aristofane troviamo varie volte il vocabolo nella sua consueta forma femminile, per esempio:

\section{Ar. Av. 565}

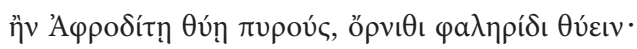

per onorare con granelli Afrodite sacrificare piselli alla falllaride. ${ }^{14}$

14 Così, enfatizzando con la triplicazione della lettera "l" un'allusione a "fallo", si cerca di riprodurre il du-

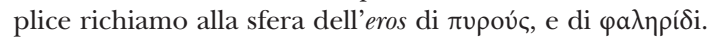


oppure nella scena iniziale delle Ecclesiazuse, quando Prassagora nella memorabile allocuzione confidenziale rivolta alla lucerna ammette che alla sua luce prova le pose di

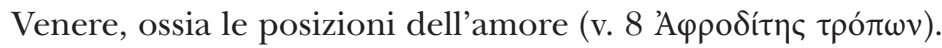

Per quel che concerne la probabile allusione alla figura mitologica dell'Ermafrodito, principio e ipostasi divina dell'unione tra i due sessi e celebrazione della fertilità, abbiamo un'antica testimonianza di questo culto, rintracciabile nell'isola dei natali di Afrodite, ossia a Cipro, dove, secondo quanto riferisce Macrobio, esisteva una statua barbuta con le fattezze di un'Afrodite maschile, denominata Afrodito da Aristofane:

\section{Macr. Sat. 3, 8, 1-3}

[...] Signum etiam eius est Cypri barbatum corpore, sed veste muliebri, cum sceptro ac natura virili: et putant eandem marem ac feminam esse. Aristophanes eam A Appóóıtov appellat... Laevinus etiam sic ait: Venerem igitur almum adorans, sive femina sive mas est, ita uti alma Noctiluca est. Philochorus quoque in Atthide eandem adfirmat esse lunam, et ei sacrificium facere viros cum veste muliebri, mulieres cum virili, quod eadem et mas aestimatur et femina. A Cipro si trova addirittura una sua statua con la barba, ma con veste femminile, dotata di scettro e statura da uomo; e ritengono che essa sia maschio e femmina. Aristofane la denomina Aphroditos, al maschile. Levino dice anche questo: adorando dunque l'almo Venere, maschio o femmina che sia, così come è l'alma Luce della notte. Anche Filocoro nell' Attide afferma che corrisponde alla luna e che a lei gli uomini celebrano sacrifici in abito muliebre, e le donne in abito maschile, dal momento che viene considerata sia maschio sia femmina.

Quella di Venere barbuta è una delle molteplici varianti con cui la dea, che presiedeva alla fertilità in tutte le sue espressioni, era venerata; solitamente portava la barba ed era maschile nella parte superiore, mentre femminile nella parte inferiore. Era chiamata per questo priapea. Simile associazione di femminile e maschile si registra a proposito della Luna. ${ }^{15}$ Si tratta qui di uno dei rari casi in cui Perotti presume nel suo lettore una conoscenza del greco, per lo meno fino al punto di riconoscere, o seguire, i tratti relativi al genere grammaticale morfologico. Ma è pure vero che dal contesto risulta particolarmente facile comprenderli.

\section{9- CC 12, 93, 16-17}

Baccar vero herba est radicis odoratae, quam nonnulli nardum rusticum falso appellant. Odor est ei cinnamomi, ex qua unguenta fieri solita testatur Aristophanes. Putabatur et contra fascinationes utilis.

15 Tale icona enigmatica sembra appartenere alle primissime forme cultuali sulla terra così come anche alla sfera pagana greco-romana. Venerata come dea madre, le cui fasi ritmiche trovano corrispondenza con i cicli di fertilità femminile, nell'ambito mediterraneo antico era tuttavia anche associata al liquido seminale e dunque celebrata nel suo duplice volto, e nell'icona ipostatica dell'androgino, misteriosa sintesi arcaica degli opposti che si rinnovano. Sul tema si veda Romanazzi (2005). Per una visione della luna come doppia, tripla, bisessuale autogenerantesi secondo varie culture, Cardamone (2006: pp. 81, 83,97 e passim). 
La baccara è un'erba dalla radice odorosa, che alcuni erroneamente denominano nardo rustico. Ha un profumo di cinnamomo, con cui, secondo la testimonianza di Aristofane, si preparavano solitamente unguenti. Si reputava efficace anche contro gli incantesimi.

Plin. $H N$ 21, 29

Baccar quoque radicis tantum odoratae est, a quibusdam nardum rusticum appellatum. Unguenta ex ea radice fieri solita apud antiquos. Aristophanes, priscae comoediae poeta, testis est; unde quidam errore falso barbaricam eam appellabant. Odor est cinnamomo proximus.

Anche la baccara ha soltanto la radice profumata e viene chiamata dal alcuni nardo rustico. Da questa radice nell'antichità si solevano preparare unguenti. Aristofane, poeta della commedia arcaica, ne è testimone; per cui erroneamente alcuni la chiamano barbarica. Il suo profumo è simile al cinnamomo.

Dopo aver dissertato intorno al nome "Bacco" e derivati, come i Baccanali, egli parla anche di questa erba odorosa; si rifà chiaramente a Plinio, come dimostra l'affinità dei termini impiegati (testatur per testis est, nardum rusticum appellant per nardum rusticum appellatum), ma per esempio in questo caso evita di spendere due parole intorno ad Aristofane, come invece aveva fatto Plinio, definendolo poeta della commedia arcaica. Il riferimento pare da riscontrarsi nel frammento Atheneo 15,41 (Ar. Th. Frg.), anche se, in contrasto con la testimonianza di Plinio e di Erotio, non sembrano necessariamente abbinate in Aristofane l'essenza della bakkaris e il profumo approntato sotto forma di unguento. ${ }^{16} \grave{E}$ vero però che in Aristofane si parla di profumi in forma di unguento, per esempio nella scena di Mirrine e Cinesia nella Lisistrata, quando al v. 944 si nomina il

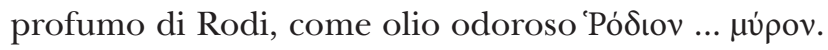

\section{0- CC 6, 402, 4-6}

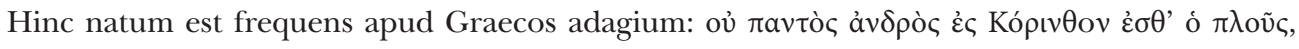
hoc est 'Non cuivis homini contingit adire Corinthum'.

Di qui è sorto il modo di dire frequente presso i Greci: non è da tutti andare a Corinto, che corrisponde al latino: ...

Gell. I, 8, 4

Ar. Fr. 902 CAF I p. 591

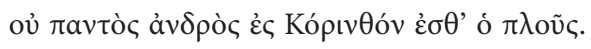

Come si vede, qui non viene citato Aristofane: la presenza del proverbio è solo indirettamente collegata con il comico; la testimonianza di Gellio indica proprio come frequens questa espressione, che presumibilmente nella commedia antica avrà suscitato il riso in quanto applicata a una situazione paradossale.

16 Greenewalt (2010), presenta alcune considerazioni al riguardo, anche in raffronto ad altre fonti. 


\section{1- CC 6, 339, 3}

Poetae eum ob corporis proceritatem poliphagum, hoc est multi cibi, et multibibum, hoc est

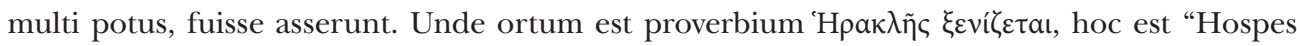
est Hercules", quotiens tarditatem alicuius significare volumus, quod ii, qui Herculem hospitio suspiciebant, tarde e convivio surgere cogebantur.

I poeti affermano che [Eracle] per la sua stazza fisica, fosse polifago, cioè mangiasse molto, e multibibo cioè bevesse molto. Per cui era sorto il proverbio "Eracle è ospitato qui", cioè "C'è ospite Eracle".

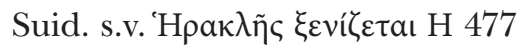

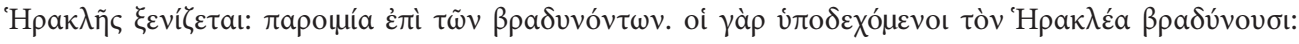

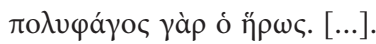

C'è ospite Eracle. Proverbio che riguarda coloro che si attardano: infatti quelli che accolgono in casa Eracle procedono con lentezza, perche l'eroe mangia molto.

Ar. Lys. 928

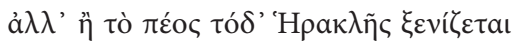

ma questo mio membro ... deve aspettare Eracle? ${ }^{17}$

Anche in questo caso, non abbiamo un rinvio esplicito ad Aristofane: sta infatti parlando del grande tributo che viene dato alla figura di Ercole come dio e, in proposito, di come egli sia anche definito "dai molti pasti" e "dalle molte bevande", in questo facendosi interprete della tradizione, che effettivamente aveva assegnato all'eroe delle dodici fatiche un posto particolare nell'ambito dell'Olimpo: quello di una figura oltre che epica e tragica anche non di rado comica (si pensi al suo ruolo di goloso all'interno dell'ambasceria dei tre dèi negli Uccelli, oppure all'Eracle di Euripide, dramma satiresco,...). Eracle

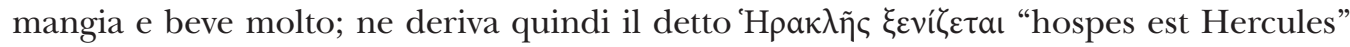
impiegato quando, per motivare il ritardo di una persona, si ipotizza ci sia in casa sua come ospite Eracle, e quindi abbia dovuto alzarsi tardi dal banchetto. Tuttavia, data la somiglianza delle espressioni utilizzate, si può trovarvi un'allusione al passo assai divertente in cui Mirrine con rocamboleschi pretesti cerca di tenere sulle spine il marito Cinesia, il quale, durante l'ennesima sua assenza strategica dal talamo, così esclama, riprendendo ironicamente in senso osceno il proverbio con riferimento al suo membro

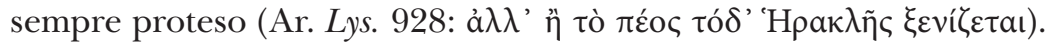

Riferisce quindi un detto della tradizione, che ritorna anche nella commedia per architettare una battuta oscena.

\section{2- CC 6, 262, 2-4}

Celebrabantur autem Proserpinae sacra Athenis nocturno tempore a solis mulieribus, crinibus passis, et ad thedarum lumina, nec fas erat alicui viro introire.

17 Paduano (1981) e Funaioli (2009) fanno invece riferimento alla tradizione (presente in Vespe, 58-60) di Eracle come insaziabile. 
Ad Atene di notte si celebravano riti sacri in onore di Proserpina, di sole donne, coi capelli scarmigliati, al fuoco delle fiaccole; agli uomini non era permesso parteciparvi.

Qui non abbiamo un effettivo rinvio al Comico; semplicemente, si può riscontrare soltanto un'affinità tra questo tema delle feste delle Tesmoforie, e lo sfondo contro cui si sviluppa la trama della commedia Tesmoforiazuse. È interessante sicuramente la presenza delle fiaccole.

\author{
Ar. Th. 101-102

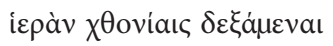

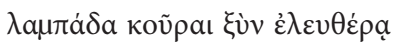 \\ con la fiaccola sacra, \\ o fanciulle, alle due dee sotterranee
}

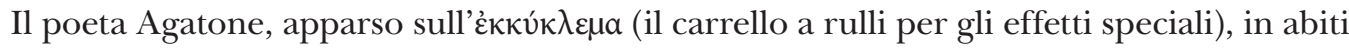
femminili, secondo la sua caricatura, intona con questi versi un peana, che viene condotto con accenti parodistici. Anche poco oltre torna il richiamo alle luci, quando si volge lo sguardo a tutta la gente che sta salendo tra il fumo delle fiaccole:

\title{
Ar. Th. 280-281
}

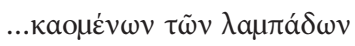

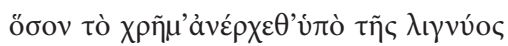 \\ quanta gente sale al fumo delle fiaccole ardenti
}

In particolare, nell'economia dei riti relativi ai misteri eleusini, si sa che si assisteva a fasi alterne di buio, in cui gli adepti erano invitati a fare l'esperienza del distacco dal mondo, della morte, per poi rinascere attraverso l'immagine della luce, rappresentata dalle fiaccole.

Anche durante le feste Scire, nelle Ecclesiazuse, Prassagora (nel monologo iniziale, vv. 1-29) parla direttamente alla lucerna come sua confidente e troviamo segnali luminosi adottati dalle compagne di Prassagora per la loro missione segreta del travestimento e dell'assemblea politica. L'allusione non può dirsi tuttavia precisa, e pare improbabile una conoscenza del testo, dal momento che l'altro elemento cui si fa cenno è la caratteristica dei capelli scarmigliati, un dato che in Aristofane non viene posto in evidenza.

\section{Perotti e le commedie di Aristofane}

Considerando l'importanza della traduzione dal greco nella vita e nella produzione del Perotti, per cui venne ingaggiato dallo stesso papa Niccolò $\mathrm{V}$, vediamo che le sue preferenze vanno ad autori come Plutarco, Polibio ed Epitteto. ${ }^{18}$ Spiccato interesse mostra

18 Troviamo una lista delle principali traduzioni da lui approntate in Oliver (1954: pp. 18-19). 
per Omero, che cita varie volte. ${ }^{19}$ In generale non sembra particolarmente orientato verso il teatro, come si può evincere dalla selezione operata nella sua prolifica attività di traduttore dal greco e anche dalle riprese soltanto indirette del corpus tragico di Eschilo, Sofocle o Euripide. Anche il ricorso ad Aristofane, che peraltro risulta essere il più considerato nell'ambito, è subordinato al ruolo di auctoritas della lingua greca; per così dire, le battute restano mute e parlano la lingua della lessicografia. La funzione ausiliaria della lingua greca nei riguardi della latina nell'impostazione generale del Cornu Copiae si riflette nel trattamento riservato ai rinvii alla commedia e può in parte spiegare la natura di queste citazioni, generalmente di seconda mano, come informazioni incontrate nel corso di personali letture, e spesso accompagnate da una traduzione. ${ }^{20}$ Del comico antico vengono infatti selezionati passi che presentino una rilevanza semantica; il riportare brani dalle commedie appare giustificato dalla presenza in essi di connotazioni relative alle accezioni dei vocaboli e ai loro usi nella lingua viva.

In effetti, Aristofane nella sua amplissima gamma di sfumature di comicità contempla anche il tipo di battuta che scaturisce direttamente dall'analisi dei vocaboli: o perché la satira si costruisce su qualche sottigliezza lessicale, o perché si attua un gioco in cui ha luogo un fraintendimento semantico di un termine, o ancora per esigenze parodistiche per cui si storpia un vocabolo, insomma, non è raro trovare in Aristofane battute il cui nucleo impegni il nocciolo semantico di un termine (basti pensare alla famosissima gaffe di Egeloco, messo in ridicolo da Aristofane), ${ }^{21}$ che aveva trasformato una battuta tragica in comica, attraverso la semplice pronuncia di $\gamma a \lambda \eta \dot{v}$ ” ("bonaccia”) come $\gamma a \lambda \tilde{\eta} v$ ("gatta”). Perotti individua le sottigliezze su cui scorre la battuta e le reimpiega con intendimento lessicografico: le ridondanze, le sfumature, i sinonimi su cui il comico greco aveva costruito le sue strategie, ritornano in lui per spiegare i contenuti e gli usi di determinati vocaboli.

Mostra di avere ben presente il futuro lettore, anzi il suo intreccio intertestuale ne appare condizionato, in quanto alcune scelte si direbbero motivate essenzialmente dal tipo di pubblico che egli presume davanti alle sue pagine. Il suo Aristofane è un'autorità il cui nome è prestigioso, sì, ma appartenente a un passato assai lontano di cui si sono perse le precise coordinate; egli sente il bisogno di presentare il Comico come non noto e, considerando l'epoca, il suo timore risulta realistico, specie tenendo conto che alcune commedie saranno edite per la prima volta soltanto nel Cinquecento.

L'assenza di una sia pur minima contestualizzazione dipende in parte anche dalle sue limitate esigenze di lessicografo: se è vero che per comprendere in profondità l'accezione di un termine la semantica ritiene imprescindibile il contesto ${ }^{22}$ è pur vero che nei

19 Charlet (2000: pp. 55-64).

20 Ritenuta la lingua da cui il latino derivava, quindi termine di riferimento fondamentale nella ricerca delle origini; cfr. Pade (2002: pp. 18-19; 2004); più in generale, Tavoni (1986). Sullo stile del Perotti si vedano i seguenti lavori: Stok (1993); Abbamonte (1998); Jocelyn (1990: pp. 99-111); Berti (1998); Volpe Cacciatore (2009).

21 Ran. 303-304.

22 Come già evidenziato da Gottlob Frege nelle sue considerazioni, secondo cui la parola non può avere un significato se non alla luce di un enunciato: Frege (1965). 
singoli casi può risultare sufficiente ai fini della specifica indagine il significato immediatamente percepibile. Mentre pensa che il lettore possa seguirlo nella forma del greco e, almeno, nella sua lettura, non lo reputa invece capace di tradurre, forse perché il significato costituisce il punto cruciale nell'ambito di una ricerca di ordine lessicografico. Le sue traduzioni sono puntuali ma essenziali, non lasciano trasparire una particolare consonanza, o gradimento alla lettura, anche se spesso, trattandosi di battute comiche, il coinvolgimento sarebbe facile. L'unica annotazione che lasci immaginare un apprezzamento da parte dell'umanista è, come si diceva, quel poeta facetissimus che, però, non è suo, ma ripreso da Plinio, dunque sembra piuttosto rispondere a un'esigenza didascalica di introduzione del passo.

Quanto appare giustificato e pertinente nel Cornu Copiae il ricorso ad Aristofane?

Esaminiamo alcune tipologie di ripresa. Il caso di scandix è molto interessante perché si configura, già nella fonte, fornito di un carattere semantico. L'intento di Aristofane è comico, ma la comicità si gestisce proprio sul filo della distinzione tra significati di due termini. Direi di più: è l'indicazione pliniana a dare lo spessore semantico di questa battuta che altrimenti nella diretta lettura della battuta teatrale sarebbe potuto sfuggire.

Il commento fornito per parochos appare a sé stante perché viene evocata una scena, che viene trasmessa, per così dire, in differita, in quanto, in occasione del matrimonio tra l'eroe Pistetero e Regina, sigillo garante di una nuova èra, si suggella l'atmosfera attraverso un precedente che ha proprio la funzione di elevare al rango divino questa situazione promiscua polirazziale e poligenetica: ossia le nozze di Zeus ed Era che avevano vantato Eros in qualità di paraninfo.

Egli eredita tale rievocazione delle nozze Zeus-Era pari pari, senza nulla aggiungere al testo originale, ma puntualmente vi appone una traduzione latina; la scena appare assai efficace, come un modo più animato, rispetto a una semplice definizione, per illustrare il sostantivo, che, portando al suo interno un verbo, può dirsi la cristallizzazione di un mestiere, quello del parochos, cosa che invece non rimane altrettanto trasparente nel termine paraninfo. La formula poetica, che ha tutta l'aria di essere a sua volta ereditata da espressioni rituali epitalamiche, è solo apparentemente ridondante in quanto le ali dorate di Eros sono strettamente connesse al suo volo e dunque al suo guidare con le redini il carro nuziale.

In conclusione, generalmente deriva la commedia di Aristofane dalle sue fonti e opera con due obiettivi, quelli di renderla utile all'indagine e comprensibile al suo lettore. Non si evince una simpatia speciale, né il desiderio di trasmettere qualcosa di più intorno all'antico modo di fare commedia. Soltanto nel caso del Pluto possiamo avanzare un'ipotesi in merito alla conoscenza diretta di una parte del testo. ${ }^{23}$

23 Tra i numerosi manoscritti greci posseduti dal Bessarione compariva il Marciano 474 contenente in papyro, comoediae septem. Sulle sorti della biblioteca bessariana, primo nucleo dell'attuale Marciana, come noto, si legga Labowsky (1979), mentre sui rapporti del manoscritto con la prima edizione a stampa e il lavoro condotto dal Musuro, Canfora (2016), specie nella sezione dedicata alla cronologia del Pluto e delle Ecclesiazuse. 


\section{Perotti e il Pluto}

Se dagli Uccelli Perotti estrae una scena, ${ }^{24}$ dagli Acarnesi una distinzione semantica, dalle Rane un ritratto parodistico, dalle Tesmoforiazuse, forse, la generale atmosfera di mistero, è solo nel caso del Pluto che, attraverso i suoi rinvii, sembra di entrare per un momento nella commedia. Anche perché egli inserisce il passo greco della citazione, dopo aver fornito indirettamente, per così dire, la scenografia per il suo antefatto: egli sta infatti dilungandosi sul tripode e sul tempio di Apollo, descrivendo con generosità di dettagli il panorama mozzafiato delle cime e dei precipizi del Parnaso, evocando la sacralità del luogo, quasi costruendo le basi per l'imminente dissacrazione che verrà operata dalle battute del servo. Infatti la scena iniziale del Pluto, che rievoca la salita e la domanda all'oracolo delfico di Apollo, è interamente gestita sulla contrapposizione a effetto tra sacralità e assurdo, in un mondo capovolto: quello che per gli altri (compreso il padrone Cremilo) è solitamente luogo di grazie, il santuario, per il servo Carione appare luogo di disgrazie; il dio che è considerato medico sembra piuttosto aver rimandato pazzo il padrone, quelli che solitamente dovrebbero fare da guida ai ciechi stanno qui seguendo un cieco...!

\section{Ar. $P l .8-14$}

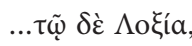

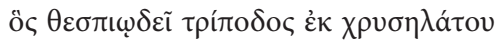

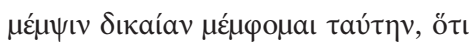

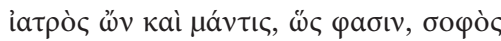

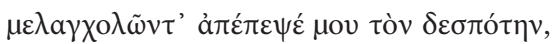

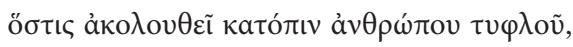

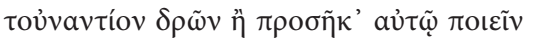

ma all'Ambiguo Apollo,

che sforna vaticini dal tripode aureo,

muovo questo giustificato rimprovero,

che, con la sua fama di alto medico e vate,

mi ha rimandato fuori di senno il mio padrone,

che sta andando dietro a un cieco, contrariamente

a quanto si dovrebbe fare

Tale ripresa dal Pluto di Aristofane acquista significato anche per altri motivi, in quanto, dopo aver presentato l'argomento relativo alle profezie delfiche ed esemplificato con il rinvio a un passo del Pluto, aggiunge un altro verso, sempre tratto da quella commedia, ma poco più oltre, limitandosi, per questo secondo, a spiegare la voce stemmata "infule"

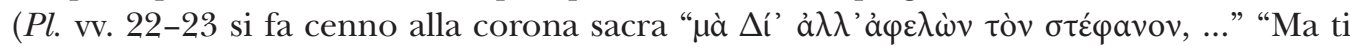
leverò la ghirlanda”). L'impressione che comunica l'umanista è quella di aver proceduto

24 D'altra parte, non si ricorda di Tereo, l'upupa aristofanea a capo del mondo degli Uccelli, parlando dell'upupa in CC 2, 705, 9-13. 
direttamente nella lettura, anche perché non pone la traduzione e non è riconoscibile la medesima fonte del primo passo; anche per la citazione della formula greca $\lambda \tilde{\eta} \rho \mathrm{v}$ $\lambda$ $\rho \varepsilon \tilde{\varsigma} \varsigma$, parimenti ripresa dal Pluto, non sente il bisogno di tradurre l'espressione che forse derivava dalla Suda o leggeva direttamente nell'originale (la traduzione del Bruni, disponibile per la prima parte, vv. 1-269, non corrisponde a questa: Bruni scriveva responsa qui dat ex tripode volvens aureo). ${ }^{25}$

L'ipotesi che Perotti leggesse direttamente il testo aristofaneo non trova ancora al momento riscontro in un manoscritto da lui annotato o posseduto; ma la sua probabilità, quale si è delineata attraverso la lettura dei passi greci di ispirazione aristofanea presenti nel Cornu Copiae, troverebbe corrispondenza nel percorso di studi e nel contesto culturale in cui scriveva l'umanista. Mi riferisco al suo maestro Vittorino. Come ricorda Z. De Cesare. ${ }^{26}$ Giovanni Tortelli era stato tra gli allievi di Vittorino da Feltre nel periodo tra il 1423 e il 1433, come possiamo ricavare dalla lettera dedicatoria della Romuli vita di Plutarco dove parla di Victorini praeceptoris mei, viri sane sapientissimi. E proprio Tortelli fu uno dei primi a interessarsi alla commedia antica all'inizio del Quattrocento, e, al ritorno da Costantinopoli e Ferrara, a dare avvio alla traduzione del Pluto in compagnia di Leonardo Bruni, come si ricorda sotto la voce "prologus" del suo De Orthographia. ${ }^{27}$ Non è infatti un mistero che il maestro Feltrense giudicasse importante per la formazione dei suoi allievi un'ampia collezione di autori greci: e che anche la lettura di Aristofane fosse valida, sia pure in una prospettiva di edificazione morale, ossia interpretandone l'autore come "persecutore dei vizi", secondo quanto riferito da Bartolomeo Platina nella sua Vita ${ }^{28}$ oltre che per la sua eleganza stilistica. D'altra parte, anche lo studio condotto dalla Cortesi sul patrimonio librario in uso nella Ca' Zoiosa dimostra che il comico antico non mancava. ${ }^{29}$ In tal senso, se l'ambito dell'educazione vittoriniana può aver incoraggiato l'interesse nei confronti dei testi aristofanei, può anche aver influenzato il tipo di lettura della commedia, non in quanto testo teatrale, ma piuttosto morale o soltano formale-letterario. Entrambi, il Tortelli per una questione legata alla struttura della commedia antica, il Perotti per sporadiche esigenze lessicografiche, senza che questo implicasse una più ampia riflessione sullo stile aristofaneo, leggono un Aristofane diverso dall'originale, o almeno dallo spirito dell'originale. Analogamente, alla fine del Quattrocento il giovane Gerolamo Amaseo nelle sue peregrinazioni in cerca di una scuola di greco, si imbatte in un Aristofane che è fondamentalmente quello della pratica di scrittura elementare. ${ }^{30}$ E lo stesso Leonardo Bruni con la sua traduzione parziale del Pluto, si preoccupava

25 Bruni (1965: p. 5).

26 De Cesare (2006: p. 110).

27 Anche in quel caso l'intento era solo lessicografico. Lo ricorda così: ut ex Aristophane libet latine versiculos aliquos referre quos Leonardus arretinus delitiae nostrae mecum olim ludens interpraetatus est. Nam in prima comoedia inducit Aristophanes Chremetem virum bonum caeterum inopem...

28 Biasuz (1948). Sui metodi impiegati da Vittorino, Garin (1949: pp. 165-178); Molti contributi sono stati dedicati al maestro nell'occasione del convegno del 1979, e raccolti negli atti: Giannetto (2012); sul rilievo dato nella filosofia della scuola all'esercizio del corpo si è soffermato di recente R. Campa (2017).

29 Cortesi (1965: pp. 77-114, 92).

30 Radif (2015). 
principalmente di mostrare quale fosse il genere della commedia antica, specialmente posta a confronto con la tradizione latina. L'apprezzamento della vena più specifica aristofanea, satirica e politica, doveva invece essere alla base dell'opera perduta realizzata da Niccolò Machiavelli attorno al 1504, dove il testo base delle Nuvole diveniva pretesto per una scena fiorentina a lui contemporanea. ${ }^{31}$

$\grave{\mathrm{E}}$ forse dunque possibile aggiungere alle vaste conoscenze del Sassoferratese questa commedia aristofanea (che già dall'inizio del secolo XV era stata ripresa e tradotta parzialmente), almeno la scena iniziale al ritorno dal santuario di Apollo.

\section{Bibliografia}

Abbamonte, G. (1998). Le ultime ricerche sull'attività lessicografica di N. Perotti. Roma nel Rinascimento, $45-58$.

Abbamonte, G. (Ed.). (2011). La battaglia nel Rinascimento meridionale: moduli narrativi tra parole e immagini. Roma: Viella.

Amigues, S. (1988). Quelques légumes de disette chez Aristophane et Plutarque. Journal des Savants (3-4), 157-171.

André, J. (1985). Les noms de plantes dans la Rome antique. Paris: Les Belles Lettres.

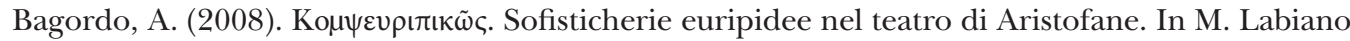
Ilundain, \& A. Melero (Eds.), Retórica y sofística en el teatro griego antiguo. Actas de la Jornada de Teatro Griego. Valencia, 12-13 diciembre de 2008 (unpublished) [retrieved 24.04.2019 from https:// www.academia.edu/30104924/\%CE\%9A\%CE\%9F\%CE\%9C\%CE\%A8\%CE\%95\%CE\%A5\%CE\% A1\%CE\%99\%CE\%A0\%CE\%99\%CE\%9A\%CE\%A9\%CE\%A3_sofisticherie_euripidee_nel_teatro_ di_Aristofane_paper_held_at_the_Jornada_de_Teatro_Griego._Ret\%C3\%B3rica_y_sof\%C3\%ADstica_en_el_teatro_griego_antiguo_Univ._Val\%C3\%A8ncia_12_dec_2008_unpublished].

Balard, M. (2004). Costantinopoli nella prima metà del Quattrocento. Medioevo Greco, 4, 7-17.

Berti, E. (1983). Ancora sulla versione del Critone di Rinuccio Aretino. Studi Classici e Orientali, 33, 119-131.

Berti, E. (1998). Manuele Crisolora, Plutarco e l'avviamento delle traduzioni umanistiche. Fontes, $1,81-99$.

Berti, E. (2006). Luciano di Samosata, Caronte, Timone. Le prime traduzioni. Firenze: Sismel.

Berti, E., \& Carosini, A. (1983). Il Critone latino di Leonardo Bruni e di Rinuccio Aretino. Firenze: Olschki.

Biasuz, G. (1948). Vita di Vittorio da Feltre (Victorini Feltrensis vita a Bartholomaeo Platina edita). Padova: Ed. Liviana.

Bruni, L. (1965). Versione del Pluto di Aristofane: di Leonardo Bruni (ed. E. Cecchini, \& M. Cecchini). Firenze: Sansoni.

Campa, R. (2017). La cura del corpo nella pedagogia umanistica di Vittorino da Feltre. In P. Prüfer (Ed.), Cztowiek sam dla siebie wyzwaniem (pp. 153-173). Gorzów Wielkopolski: Wydawnictwo Naukowe Akademii im. Jakuba z Paradyża.

31 Radif (2010) 
Canfora, L. (2016). La crisi dell' utopia: Aristofane contro Platone. Bari: Laterza.

Cardamone, A. (2006). In traccia di luna. Mitologie lunari tra oralità e scrittura. Cosenza: Pellegrini Editore.

Cecchini, E., \& Cassio, A. C. (1972). Due contributi sulla traduzione di Leonardo Bruni del Pluto di Aristofane. Giornale Italiano di Filologia, 24, 472-482.

Cessi, R. (1913). La giovinezza di Pietro Tomasi. Athenaeum, 1, 129-161.

Charlet, J.-L. (Ed.). (1989-2001). Nicolai Perotti: Cornv copiae seu linguae Latinae commentarii. Sassoferrato: Istituto Internazionale di Studi Piceni.

Charlet, J.-L. (1990). Sur dix citations d'auteurs antiques dans le Cornu copiae de N. Perotti: remarques méthodologiques. Studi Umanistici Piceni, 10, 41-47.

Charlet, J.-L. (1994). Tortelli et un certain Parthenius'. Studi Umanistici Piceni, 14, 21-26.

Charlet, J.-L. (2000). Homère chez deux lexicographes humanistes, G. Tortelli (De orthographia) et N. Perotti (Cornu copiae). In F. Montanari, \& S. Pittaluga (Eds.), Posthomerica, II: tradizioni omeriche dall'Antichità al Rinascimento (pp. 55-64). Genova: Dipartimento di archeologia, filologia classica e loro tradizioni.

Charlet, J.-L. (2009). État présent des études sur Niccolò Perotti (1993-2008). Humanistica, 2, 119-130.

Charlet, J.-L. (2011a). Niccolò Perotti, humaniste du Quattrocento: Bibliographie critique. Rencessanceforum. Tidsskrift for rencessanceforskning, 7, 1-72.

Charlet, J.-L. (2011b). La réception de l'Histoire Naturelle de Pline dans le Cornu copiae de N. Perotti. Archives Internationales d'Histoire des Sciences, 61, 237-248.

Charlet, J.-L. (2013). La culture grecque de Niccolò Perotti. Cahiers de recherches médiévales et humanistes, 25, 259-280.

Cortesi, M. (1965). Libri e vicende di Vittorino da Feltre. Italia Medioeva e Umanistica, 8, 77-114.

Cortesi, M. (1995). La tecnica del tradurre presso gli umanisti. In C. Leonardi, \& B. Munk Olsen (Eds.), The Classical Tradition in the Middle Ages and the Renaissance. Proceedings of the First European Workshop on «The Reception of Classical Texts» (Florence, Certosa del Galluzzo, 26-27 June 1992) (pp. 143-168). Spoleto: Centro Italiano di Studi sull'Alto Medioevo.

De Cesare, Z. (2006). Le traduzioni latine del Pluto di Aristofane nel XV secolo: Rinuccio di Arezzo, Leonardo Bruni e Pietro da Montagnana (Diss. thesis, University of Parma) [retrieved 24.04.2019 from https://www.google.com/search?q=Le+traduzioni+latine+del+Pluto+di+Aristofane ${ }^{+-}$ nel+XV+secolo $\% 3 \mathrm{~A}+\mathrm{Rinuccio}+\mathrm{di}+\mathrm{Arezzo} \% 2 \mathrm{C}+\mathrm{Leonardo}+\mathrm{Bruni}+\mathrm{e}+\mathrm{Pietro}+\mathrm{da}+$ Montagnana\&rlz=1C1CAFA_enCZ763CZ763\&oq $=\mathrm{Le}+$ traduzioni+latine + del+Pluto+di+Aristofane + nel+XV+secolo $\% 3 \mathrm{~A}+$ Rinuccio $+\mathrm{di}+$ Arezzo $\% 2 \mathrm{C}+\mathrm{Leonardo}+$ Bruni $+\mathrm{e}+$ Pietro $+\mathrm{da}+$ Montagnana\&aqs $=$ chr ome..69i57j69i60.416j0j7\&sourceid=chrome\&ie=UTF8].

Frege, G. (1965). I fondamenti dell'aritmetica. Un'indagine logico-matematica sul concetto di numero (transl. G. Geymonat, \& C. Mangione). In Idem, Logica e Aritmetica (pp. 207-349). Torino: Bollati Boringhieri (original title Die Grundlagen der Arithmetik. Eine logisch-mathematische Untersuchung über den Begriff der Zahl. Breslau: Koebner1884).

Funaioli, M. P. (Ed.). (2009). Aristofane: Lisistrata. Siena: Barbera.

Garin, E. (1949). L'educazione umanistica in Italia. Bari: Laterza.

Giannetto, N. (Ed.). (2012; 1981). Vittorino da Feltre e la sua scuola. Umanesimo, pedagogia, arti. Atti del Convegno di studi promosso dalla Fondazione Giorgio Cini dai Comuni di Feltre e di Mantova 
e dall'Accademia Virgiliana di Mantova nel sesto centenario della nascita di Vittorino da Feltre, Venezia-Feltre-Mantova, 9-11 novembre 1979. Firenze: Olschki.

Greenewalt, C. H. (2010). Lydian Cosmetics / Lidya Kozmetiği. In N. D. Cahill (Ed.), The Lydians and Their World (pp. 201-216). Istanbul: Yap1 Kredi Yayınlar1 [retrieved 24.04.2019 from http:// sardisexpedition.org/en/essays/latw-greenewalt-lydian-cosmetics].

Jocelyn, H. D. (1990). The Sources of the Cornu copiae of N. Perotti and their Integrity: Some Methodological Remarks. In S. Prete (Ed.), Memores tui. Studi di letteratura classica ed umanistica in onore di Marcello Vitaletti (pp. 99-111). Sassoferrato: Istituto Internazionale Studi Piceni.

Labowsky, L. (1979). Bessarion's Library and the Biblioteca Marciana. Roma: Edizioni di Storia e Letteratura.

Lockwood, D. P. (1909). Aristophanes in the XVth Century. Transactions and Proceedings of the American Philological Association, 40, LVI.

Lockwood, D. P. (1913). De Rinucio Aretino Graecarum Litterarum Interprete. Harvard Studies in Classical Philology, 24, 51-109.

Lockwood, D. P. (1918). Two Thousand Years of Latin Translation from Greek. Transactions and Proceedings of the American Philological Association, 49, 115-129.

Lockwood, D. P. (1931). Leonardo Bruni's Translation of Act I of the Plutus of Aristophanes. In G. D. Hadzsits (Ed.), Classical studies in honor of F. C. Rolfe (pp. 163-172). Philadelphia: University of Philadelphia Press.

Lockwood, D. P. (1938). De domo Rinucii. In L. Webber Jones (Ed.), Studies in honor of E. K. Rand (pp. 177-191). New York: Butler Hall.

Ludwig, W. (1975). Die Fabula Penia des Rinucius Aretinus. München: Fink.

Mattioli, P. A. (1744). Discorsi di M. Pietro Andrea Mattioli sanese, medico cesareo, ne' sei libri di Pedacio Dioscoride anazarbeo della materia medicinale: colle figure delle piante, ed animali cavate dal naturale. Con due tavole copiosissime: l' una intorno a ciò, che in tutta l' opera si contiene: e l'altra della cura di tutte le infermità del corpo umano. Venezia: Niccolò Pezzana.

Mercati, G. (1946). Di Giovanni Simeonachis protopapa di Candia. In Idem, Miscellanea Giovanni Mercati (Vol. III; pp. 312- 341). Città del Vaticano: Biblioteca Apostolica Vaticana.

Oliver, R. (1954). Niccolo Perotti's Version of the Enchiridion of Epictetus (with an introduction and a list of Perotti's Writings). Urbana: University of Illinois Press.

Pade, M. (2002). Il greco del Perotti. Studi Umanistici Piceni, 22, 17-28, esp. 18-19.

Pade, M. (2004). I manoscritti del Perotti (1429/1430-1480) e il materiale utilizzato per il Cornu copiae. In J. Hamesse, \& M. Fattori (Eds.), Lexiques et glossaires philosophiques de la Renaissance (pp. 73-95). Turnhout: Brepols.

Paduano, G. (Ed.). (1981). Aristofane: Lisistrata. Milano: Rizzoli.

Pillolla, M. P. (1993). Rinucius Aretinus: Fabulae Aesopicae (Favolisti Latini Medievali e Umanistici, IV). Genova: Dipartimento di archeologia, filologia classica e loro tradizioni.

Polito, A. (2011). L'erba che ricorda le unghie del diavolo. In Fondazione Terra d'Otranto [retrieved 24.04.2019 from http://www.fondazioneterradotranto.it/2011/01/25/lerba-che-ricorda-le-unghia-del-diavolo/].

Prete, S. (1980). L'umanista Niccoló Perotti. Sassoferrato: Istituto internazionale di studi piceni.

Prete, S. (1986). Possibilità di ricerche nel Cornucopiae di N. Perotti. Nuovi Studi Fanesi, 1, 51-80.

Prete, S. (1990), Le citazioni delle commedie di Terenzio nel Cornu copiae di N. Perotti. In Idem 
(Ed.), Memores tui (pp.137-153). Sassoferrato: Istituto Internazionale Studi Piceni.

Radif, L. (2010). Le Maschere di Machiavelli. Imperia: Ennepilibri.

Radif, L. (Ed.). (2011). Rinuccio Aretino: Penia (praef. A. Stäuble). Firenze: Franco Cesati.

Radif, L. (2015). L'Aristofane elementare della lettera di Girolamo Amaseo al fratello Gregorio.

In L. Secchi Tarugi (Ed.), Pio II nell'epistolografia del Rinascimento. Atti del XXV Congresso di Studi Umanistici Istituto Francesco Petrarca, Chianciano Terme-Pienza, 18-20 luglio 2013 (pp. 351-358). Firenze: Franco Cesati.

Romanazzi, A. (2005). Guida alla dea madre in Italia: itinerari fra culti e tradizioni popolari. Roma: Venexia.

Rossi, V. (1933). Il Quattrocento. Milano: Vallardi.

Sabbadini, R. (1922). Il metodo degli umanisti. Firenze: Le Monnier.

Stäuble, A. (1976). La commedia umanistica: bilancio e prospettive. Maia, 28, 255-265.

Stäuble, A. (1985). Rassegna bibliografica. Teatro del Quattrocento. Giornale Storico della Letteratura Italiana, 162, 276-288.

Stok, F. (1993). Le laboratoire lexicographique de N. Perotti. Vichiana, 4, 100-110 (repr. in Stok, F. Studi sul Cornu Copiae di Niccolò Perotti. Pisa: ETS 2002).

Tavoni, M. (1986). On the Renaissance Idea that Latin Derives from Greek. Annali della Scuola Normale Superiore di Pisa, 16, 205-238.

Viti, P. (Ed.). (2004). Leonardo Bruni: Sulla perfetta traduzione. Napoli: Liguori.

Volpe Cacciatore, P. (2009). Plutarco nelle traduzioni latine di età umanistica. Napoli: M. D'Auria.

Weiss, R. (1964). Un umanista antiquario, Cristoforo Buondelmonti. Lettere Italiane, 16, 105-116.

Weiss, R. (1969). La scoperta dell'antichità classica nel Rinascimento (transl. M. T. Bindella). Padova:

Antenore (original title The Renaissance Discovery of Classical Antiquity. Oxford: Blackwell 1969).

Woodward, W. H. (1897). Vittorino da Feltre and Other Humanist Educators. Cambridge: University Press.

Ludovica Radif, Ph.D. / ludovica.radif@upol.cz

Department of Classical Philology

Palacký University in Olomouc, Philosophical Faculty

Na Hradě 5, 77180 Olomouc, Czech Republic

This work can be used in accordance with the Creative Commons BY-SA 4.0 International license terms and conditions (https://creativecommons.org/licenses/by-sa/4.0/legalcode). This does not apply to works or elements (such as image or photographs) that are used in the work under a contractual license or exception or limitation to relevant rights. 